\title{
Coupled tension-torsion vibration of repetitive beam-like structures
}

\author{
N.G. Stephen*, Y. Zhang \\ School of Engineering Sciences, Mechanical Engineering, University of Southampton, Highfield, Southampton SO17 1BJ, UK
}

Received 21 April 2005; received in revised form 22 September 2005; accepted 29 September 2005

Available online 13 December 2005

\begin{abstract}
Equivalent continuum stiffness properties, derived from the eigenanalysis of a single cell of a planar beam-like repetitive structure, have previously been employed within well-known dynamic theories, such as Euler-Bernoulli and Timoshenko for flexural vibration, suitably modified, to predict natural frequencies of vibration. Here the approach is applied to two structure types that exhibit tension-torsion coupling. The first is modelled on a NASA deployable structure with a crosssection of equilateral triangular form, but with asymmetric triangulation on the faces. The second is a related, more symmetric, structure but with pre-twist. The simplest tension-torsion dynamic theory due to Di Prima is employed, and this is extended to more general end conditions. This combined periodic structure/substitute continuum approach provides excellent agreement with predictions from the finite element method, especially for the lower modes of vibration; typically, agreement is within $\pm 1 \%$ for the lowest 8-10 natural frequencies for the longer, 30-cell structures considered here, the majority of these being torsional modes, and within $\pm 1 \%$ for the lowest $4-5$ modes for the shorter, ten-cell, structures. This level of accuracy is attainable so long as a single wavelength spans 2-3 cells of the repetitive structure.
\end{abstract}

(C) 2005 Elsevier Ltd. All rights reserved.

\section{Introduction}

Repetitive, or periodic, structures consist of a basic cell that repeats in one, two, or three dimensions. Rail track supported on equally spaced sleepers, and a honeycomb sandwich panel, are examples of onedimensional (beam-like) and two-dimensional (plate-like) repetitive structures, respectively. Component manufacture and assembly of such structures are also repetitive, so they often represent a cost effective design solution in a variety of mechanical, civil and aerospace applications. In a 1988 review, Noor [1] proposed four classes of approach to their analysis; these are:

(a) Direct method in which the complete structure is analysed as a system of discrete members by the finite element method (FEM).

(b) Direct field method in which the displacements on either side of the repeating cell are related by finite difference equations; this approach was developed extensively by Dean [2] and more recently by Renton [3].

*Corresponding author. Tel.: + 44(0)23 80592359; fax: + 44(0)2380593230.

E-mail address: ngs@soton.ac.uk (N.G. Stephen). 


$\begin{array}{llll}\text { Nomenclature } & m, M_{x} & \begin{array}{l}\text { mass (including per unit length), twisting } \\ \text { moment }\end{array} \\ A & \text { cross-sectional area } & n & \text { integer } \\ d, \mathbf{d} \quad \text { rod diameter, nodal displacement vector } & r & \text { radius of rod } \\ E & \text { Young's modulus of elasticity } & T_{x}, T & \text { tensile force, kinetic energy } \\ \mathbf{F} & \text { nodal force vector } & u, U & \text { extension, constant, strain energy } \\ G, \mathbf{G} \quad \text { shear modulus, transfer matrix } & x, y, z & \text { Cartesian coordinates } \\ \mathrm{i}, I \quad & \sqrt{-1}, \text { moment of inertia (including per } & \alpha & \text { angle of pre-twist per cell } \\ & \text { unit length) } & \lambda & \text { root of characteristic equation } \\ J & \text { torsion constant } & \theta, \Theta \quad \text { angle of rotation, constant } \\ K_{\mathrm{tt}}, \mathbf{K} \quad \text { tension-torsion coupling coefficient, } & \rho & \text { mass density } \\ & \text { stiffness matrix } & \omega & \text { radian frequency } \\ l, L & \text { length of rod, beam } & & \end{array}$

(c) Periodic structure approach, which typically employs a transfer matrix relating a state vector (consisting of nodal displacement and force components) on either side of the generic cell. For dynamic analysis, translational symmetry or, equivalently, Bloch's theorem leads to an eigenvalue problem for propagation constants or frequencies in the dynamic case; this highly developed approach has been applied successfully to both one- and two-dimensional structure, [4-11]. For the static case, which is less well developed, non-unity eigenvalues describe the decay of self-equilibrated end loading, as anticipated by Saint-Venant's principle. Multiple unity eigenvalues pertain to transmitting loads such as tension, torsion, bending moment and shearing force, together with rigid body displacements and rotations. Straight and curved planar, and pre-twisted, beam-like repetitive structures have been considered by Stephen and co-workers [12-15].

(d) Substitute continuum approach in which the original structure is replaced by a continuum whose properties are in some sense equivalent.

Of the first three, approach (a) is the least efficient computationally, as the periodicity of the structure is not exploited. Approach (c) leads to the familiar mathematics and considerable body of literature associated with an eigenvalue problem. In principle, the behaviour of a complete structure can be determined through the analysis of a single repeating cell, together with boundary conditions if the structure is not of infinite extent, with no approximations above and beyond those that would have been employed in (a).

Different approaches are also employed for dynamic analysis according to whether one is interested in the high, medium or low frequency ranges. Statistical energy analysis (SEA) is often employed for the high frequency range, when approach (a) may not be feasible due to the excessive number of degrees of freedom necessary to capture the short wavelength behaviour [16]. The mid-frequency range, for which the wavelengths are comparable to the lengths of the individual rods or struts that comprise the structure, has been neglected by engineers, according to Machens and Dyer [17], although hybrid SEA/FEM schemes have been introduced [18]. The present paper is concerned with the low frequency range, where wavelength is greater than the length of individual members, and the dominant behaviour of the structure can be described adequately in "beamlike" terms, such as flexural, extensional or torsional. Noor's approach (d) is ideally suited to this range; moreover, the relevant substitute, or equivalent continuum properties can be determined efficiently using the periodic structure approach (c). Thus, the present approach may be regarded as a combination of Noor's periodic structure (c), and substitute continuum methods (d).

For the planar structure considered in [12], the transfer matrix $\mathbf{G}$ was constructed through manipulation of the stiffness matrix $\mathbf{K}$ of a single repeating cell. The equivalent continuum properties, such as cross-sectional area $A$, second moment of area $I$, and shear coefficient $\kappa$ were derived from the eigen- and principal vectors associated with the multiple unity eigenvalues. These properties were employed subsequently [19] to determine natural frequencies of a planar beam-like structure, using well-known dynamic theories, such as those due to 
Euler-Bernoulli and Timoshenko (suitably modified), and shown to provide very good agreement with FEM predictions, so long as the semi-wavelength is greater than the length of a typical rod or strut within the cell. The present paper extends the approach to the vibration of repetitive one-dimensional (beam-like) structures that exhibit tension-torsion coupling. The static behaviour is assumed to be governed by coupled equations of the form

$$
\left[\begin{array}{l}
T_{x} \\
M_{x}
\end{array}\right]=\left[\begin{array}{ll}
E A & K_{\mathrm{tt}} \\
K_{\mathrm{tt}} & G J
\end{array}\right]\left[\begin{array}{l}
\partial u / \partial x \\
\partial \theta / \partial x
\end{array}\right]
$$

or $\mathbf{F}=\mathbf{K d}$, where the generalised force vector $\mathbf{F}$ consists of the tensile force $T_{x}$, and the torsional moment about the longitudinal $x$-axis, $M_{x}$, respectively; the displacement vector $\mathbf{d}$ consists of the rates of change of extension $u$ and rotation about the $x$-axis, $\theta$, in the $x$-direction. Within the stiffness matrix $\mathbf{K}$, the axial and torsional rigidities are $E A$ and $G J$, respectively, and these differ from those of the straight structure. Coupling strength is defined by the coefficient $K_{\mathrm{tt}}$, and is symmetric as demanded by the reciprocal theorem. Axial stiffness is, in the main, greater than torsional stiffness, although one is not comparing like with like. In turn, uncoupled natural frequencies of extensional vibration are greater than those of torsional vibration; for example, a continuum rod of circular cross-section has extensional frequencies proportional to $\sqrt{E / \rho}$, while torsional frequencies are proportional to $\sqrt{G / \rho}$, assuming identical mode number and end conditions. Tension-torsion coupling is known to occur for structures having pre-twist (see Rosen [20] for an extensive review) and has the effect of bringing their stiffness' closer together; thus pre-twist can increase torsional stiffness appreciably while decreasing axial stiffness, although not by so much. The stiffening effect of pretwist on torsional vibration appears to have been studied first by Reissner and Washizu [21], who specialised equations derived from thin shell theory to consider pre-twisted beams of narrow rectangular cross-section. Later, Di Prima [22] derived coupled equations of the same form as Eq. (1), based on Chen's helical fibre assumption for pre-twist [23]; Di Prima [22] found that frequencies of the predominantly torsional mode are increased significantly by pre-twist, while frequencies of the predominantly extensional modes are only slightly lowered. ${ }^{1}$

Motivation for the present study arose initially from the eigenanalysis of an asymmetric repetitive pinjointed structure [13], based upon a NASA deployable satellite boom structure [24], Fig. 1, which displays both tension-torsion, and bending-shear coupling. (The latter, wherein curvature in the plane of the applied bending moment is coupled with a shear deflection in the perpendicular plane, is not explored in the present work.) That study, in turn, motivated the eigenanalysis of a pre-twisted repetitive pin-jointed structure [15], Fig. 2, which again exhibits tension-torsion coupling according to Eq. (1). The two structure types considered are not unrelated: the asymmetric has a single diagonal rod in each face, consistent with the minimum triangulation requirement. In the pre-twisted structure, this is replaced by two diagonal rods placed symmetrically, each having a cross-sectional area equal to one-half of the original single diagonal rod. Thus the mass per unit length, and the rotational moment of inertia, are identical for both structures when the angle of pre-twist is zero.

The primary objective of the present work is to extend the equivalent continuum approach to the dynamic analysis of repetitive structures, as described in [19], to structures that specifically display tension-torsion coupling. A secondary objective is to extend the dynamic theory of tension-torsion coupling presented in [22] to more general end conditions. First, Hamilton's principle is employed to determine the governing dynamic equations, which are solved for a variety of boundary conditions. Determination of the equivalent continuum stiffness properties of the asymmetric and pre-twisted structures is described fully in [13,15], respectively, and is not repeated here. Inertia properties are found by elementary means. Frequency predictions are compared with those obtained from FEM, which are regarded as the benchmark for accuracy, for a variety of lengths and boundary conditions. Best agreement is achieved employing the consistent mass matrix option that, according to Desai and Abel [25], provides frequencies that are proven upper bound.

For the pre-twisted structure, attention is focussed initially on a somewhat arbitrary pre-twist of $\alpha=$ $\pi / 8\left(=22.5^{\circ}\right)$ per cell, as in [15]; however, the natural frequencies for the fundamental modes of both torsional

\footnotetext{
${ }^{1}$ Note that a missing $\beta$ in the expression for the twisting moment in Eq. (6) of [22] incorrectly suggests that coupling is not symmetric.
} 


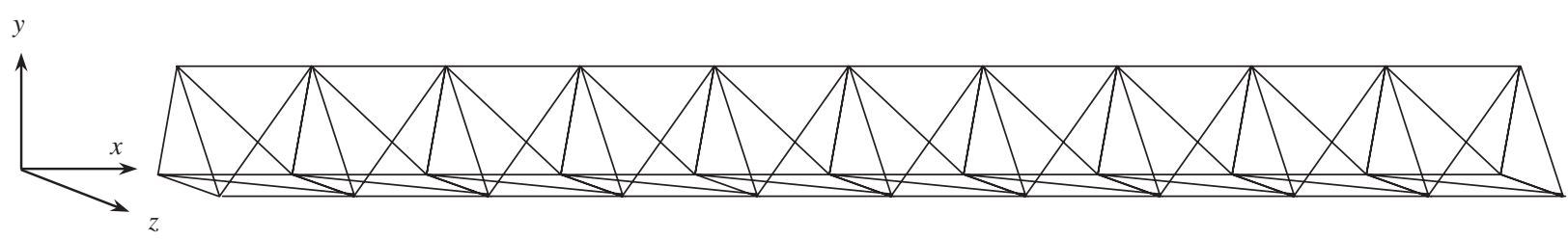

Fig. 1. A ten-cell asymmetric 3-D pin-jointed framework having equilateral triangular cross-section.

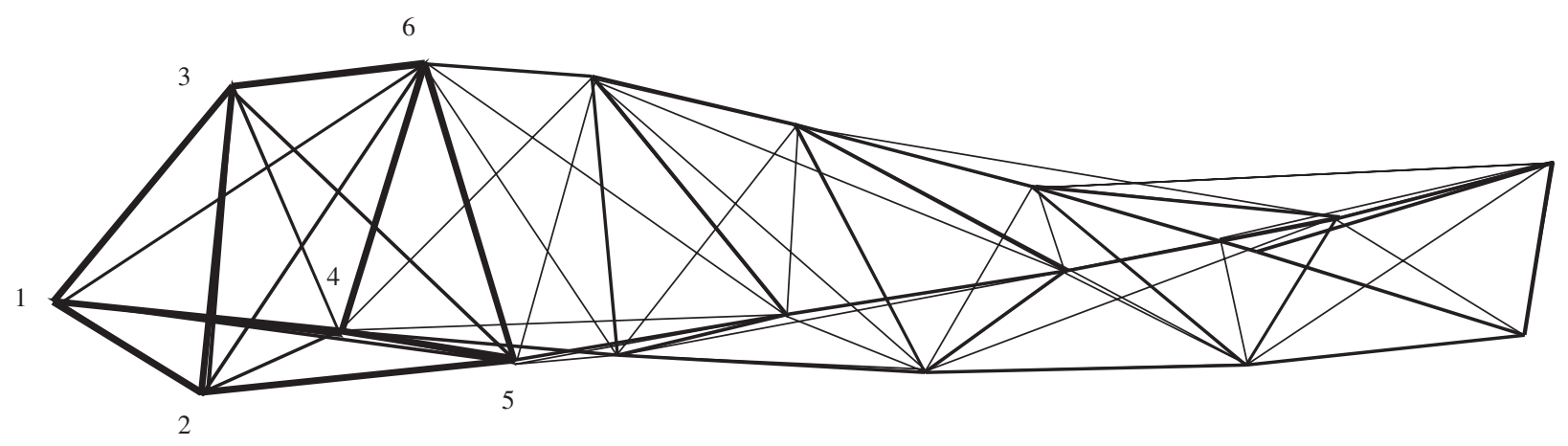

Fig. 2. A six-cell pin-jointed pre-twisted framework; the first cell is shown in bold.

and extensional modes under various boundary conditions are determined for a 10-cell structure with pre-twist angle over the range of $0-180^{\circ}$ per cell.

As with the planar structure considered in [19], the present approach provides very good agreement with the benchmark FEM predictions especially for the lowest modes of vibration.

\section{The structures and their equivalent continuum properties}

\subsection{General description}

Both structure types considered consist of circular section aluminium struts, or rods, having diameter $d=6.35 \mathrm{~mm}$ and length $l=342.8 \mathrm{~mm}$, which are pin-jointed to form a beam-like structure having an equilateral triangular cross-section; the axial length of each cell is also taken as $l$. The asymmetric structure has three horizontal rods joining cross-sectional nodes, and a single diagonal rod of length $\sqrt{2} l$ to triangulate each face of the cell; again these have diameter $d=6.35 \mathrm{~mm}$. For the pre-twisted structure, adjacent cross-sections are rotated through uniform $\alpha$ radians, initially taken as $\alpha=\pi / 8$. The longitudinal (helical) rods have length as demanded by the nodal locations, while each face of the cell now has two diagonal rods (such that the structure is symmetric for zero pre-twist angle) of the necessary length, with each having a cross-sectional area one-half of the longitudinal rods, equivalent to a diameter of $d / \sqrt{2}$. For both structures, the Young's modulus and the density are taken as $E=70 \times 10^{9} \mathrm{~N} / \mathrm{m}^{2}$ and $\rho=2700 \mathrm{~kg} / \mathrm{m}^{3}$, respectively. For the eigenanalysis, determination of the equivalent continuum stiffness properties, and also elementary calculation of the mass and inertia properties, the cross-sectional rods are regarded as being shared between adjacent cells, so they are modelled as having a diameter of $d / \sqrt{2}$.

The mass per unit length is calculated simply as the sum of the individual masses of the rods that constitute the generic cell, divided by the length of the cell. The moment of inertia per unit length of the cell is calculated as follows: first, the moments of inertia of each rod are calculated about its own centre of gravity, taken as the origin of a local coordinate system, with the local $x$-axis directed along the length of the rod, using the wellknown simple formulae $I_{y}=I_{z}=m l^{2} / 12$, and $I_{x}=m r^{2} / 2$, consistent with the length of the rods being significantly greater than the radius; however, for both structure types the moment of inertia $I_{y}\left(=I_{z}\right)$ of a typical rod is approximately 2000 times larger than $I_{x}$, so contributions from the latter are ignored. These are 
then converted to moment of inertia about the global $x$-axis, using appropriate coordinate transformations and the parallel axis theorem. Last, they are added to give the moment of inertia for the complete cell, which is then divided by the length.

\subsection{The asymmetric structure}

The stiffness matrix is, from [13]

$$
\mathbf{K}=\left[\begin{array}{ll}
E A & K_{\mathrm{tt}} \\
K_{\mathrm{tt}} & G J
\end{array}\right]=\left[\begin{array}{cc}
8.3877 \times 10^{6} & -1.7190 \times 10^{5} \\
-1.7190 \times 10^{5} & 1.7011 \times 10^{4}
\end{array}\right],
$$

where $E A, K_{\mathrm{tt}}$ and $G J$ have units $\mathrm{N}, \mathrm{Nm}$ and $\mathrm{Nm}^{2}$, respectively. The mass per unit length is $m=0.8758 \mathrm{~kg} / \mathrm{m}$, while the moment of inertia about the $x$-axis per unit length is found to be $I_{x}=2.2177 \times 10^{-2} \mathrm{~kg} \mathrm{~m}$. The negative value of the coupling coefficient $K_{\mathrm{tt}}$ is a consequence of the chosen direction for positive twisting moment and rotation.

\subsection{The pre-twisted structure}

For a pre-twist angle of $\alpha=\pi / 8$, the stiffness matrix is, from [15]

$$
\mathbf{K}=\left[\begin{array}{ll}
E A & K_{\mathrm{tt}} \\
K_{\mathrm{tt}} & G J
\end{array}\right]=\left[\begin{array}{cc}
7.8641 \times 10^{6} & -1.8578 \times 10^{5} \\
-1.8578 \times 10^{5} & 3.4099 \times 10^{4}
\end{array}\right]
$$

and the same units are employed. The mass per unit length is $m=0.8794 \mathrm{~kg} / \mathrm{m}$, while the moment of inertia about the $x$-axis per unit length is found to be $I_{x}=2.2203 \times 10^{-2} \mathrm{~kg} \mathrm{~m}$. Again, the negative coupling coefficient is a consequence of the chosen sign convention for twisting moment and rotation; in [15], moment was defined as positive when tending to undo the pre-twist, whereas Di Prima [22] defined moment as positive when the attendant rotation was in the same sense as the pre-twist, leading to a positive coupling coefficient.

\section{Continuum dynamic theory}

\subsection{Tension-torsion dynamic equations}

According to Hamilton's principle, the governing equations and boundary conditions may be generated according to

$$
\delta \int_{t_{1}}^{t_{2}}(T-U) \mathrm{d} t=0
$$

where the kinetic and strain energies are

$$
\begin{aligned}
& T=\frac{1}{2} \int_{0}^{L}\left\{m\left(\frac{\partial u}{\partial t}\right)^{2}+I_{x}\left(\frac{\partial \theta}{\partial t}\right)^{2}\right\} \mathrm{d} x, \\
& U=\frac{1}{2} \int_{0}^{L} \mathbf{d}^{\mathrm{T}} \mathbf{K d} \mathrm{d} x
\end{aligned}
$$

and the generalised stiffness matrix $\mathbf{K}$ and displacement vector $\mathbf{d}$ are given in Eq. (1); note that $L$ denotes the length of the entire structure. Performing the first variation of Eq. (4), and integrating by parts in the usual way, gives the dynamic equations

$$
E A \frac{\partial^{2} u}{\partial x^{2}}+K_{\mathrm{tt}} \frac{\partial^{2} \theta}{\partial x^{2}}=m \frac{\partial^{2} u}{\partial t^{2}},
$$




$$
G J \frac{\partial^{2} \theta}{\partial x^{2}}+K_{\mathrm{tt}} \frac{\partial^{2} u}{\partial x^{2}}=I_{x} \frac{\partial^{2} \theta}{\partial t^{2}}
$$

together with the boundary conditions:

$$
\begin{aligned}
& \text { either } E A \frac{\partial u}{\partial x}+K_{\mathrm{tt}} \frac{\partial \theta}{\partial x}=0 \text { equivalent to } T_{x}=0 \text { or } \delta u=0, u \text { is specified, } \\
& \text { either } G J \frac{\partial \theta}{\partial x}+K_{\mathrm{tt}} \frac{\partial u}{\partial x}=0 \text { equivalent to } M_{x}=0 \text { or } \delta \theta=0, \theta \text { is specified. }
\end{aligned}
$$

Separation of variables by writing

$$
u(x, t)=U(x) \sin \omega t \text { and } \theta(x, t)=\Theta(x) \sin \omega t
$$

leads to the coupled ordinary differential equations

$$
E A U^{\prime \prime}+K_{\mathrm{tt}} \Theta^{\prime \prime}+\omega^{2} m U=0, \quad G J \Theta^{\prime \prime}+K_{\mathrm{tt}} U^{\prime \prime}+\omega^{2} I_{x} \Theta=0,
$$

where prime denotes differentiation with respect to $x$. These may be uncoupled, leading to the two fourthorder equations

$$
\left(E A G J-K_{\mathrm{tt}}^{2}\right)\left[\begin{array}{l}
U^{\prime \prime \prime \prime \prime} \\
\Theta^{\prime \prime \prime \prime}
\end{array}\right]+\omega^{2}\left(G J m+E A J_{x}\right)\left[\begin{array}{l}
U^{\prime \prime} \\
\Theta^{\prime \prime}
\end{array}\right]+\omega^{4} I_{x} m\left[\begin{array}{l}
U \\
\Theta
\end{array}\right]=\mathbf{0} .
$$

Assuming axial dependence as $\exp (\mathrm{i} \lambda x)$ leads to the characteristic equation

$$
\left(E A G J-K_{\mathrm{tt}}^{2}\right) \lambda^{4}-\left(G J m+E A I_{x}\right) \omega^{2} \lambda^{2}+\omega^{4} I_{x} m=0 .
$$

The roots are

$$
\left(\frac{\lambda_{1,2}}{\omega}\right)^{2}=\frac{\left(E A I_{x}+G J m\right) \pm \sqrt{\left(E A I_{x}+G J m\right)^{2}-4 m I_{x}\left(E A G J-K_{\mathrm{tt}}^{2}\right)}}{2\left(E A G J-K_{\mathrm{tt}}^{2}\right)}
$$

or equivalently

$$
\left(\frac{\omega_{1,2}}{\lambda}\right)^{2}=\frac{\left(E A I_{x}+G J m\right) \pm \sqrt{\left(E A I_{x}+G J m\right)^{2}-4 m I_{x}\left(E A G J-K_{\mathrm{tt}}^{2}\right)}}{2 m I_{x}} .
$$

Note that the term $\left(E A G J-K_{\mathrm{tt}}^{2}\right)$ within Eqs. (14), (15) is the determinant of the stiffness matrix $\mathbf{K}$ in Eq. (1), and this must be positive, as the strain energy must be positive definite during vibration. It follows that the roots $\left(\lambda_{1} / \omega\right)^{2}$ and $\left(\lambda_{2} / \omega\right)^{2}$ in Eq. (14) are both positive, and so represent modes having a (co)sinusoidal form. Consequently, the general solutions are

$$
\left[\begin{array}{l}
U(x) \\
\Theta(x)
\end{array}\right]=\left[\begin{array}{l}
U_{1} \\
\Theta_{1}
\end{array}\right] \sin \lambda_{1} x+\left[\begin{array}{l}
U_{2} \\
\Theta_{2}
\end{array}\right] \cos \lambda_{1} x+\left[\begin{array}{c}
U_{3} \\
\Theta_{3}
\end{array}\right] \sin \lambda_{2} x+\left[\begin{array}{c}
U_{4} \\
\Theta_{4}
\end{array}\right] \cos \lambda_{2} x .
$$

The eight constants in the above are not independent, but are related as

$$
\Theta_{1}=U_{1} \frac{\omega^{2} m-E A \lambda_{1}^{2}}{K_{\mathrm{tt}} \lambda_{1}^{2}}, \quad \Theta_{2}=U_{2} \frac{\omega^{2} m-E A \lambda_{1}^{2}}{K_{\mathrm{tt}} \lambda_{1}^{2}}, \quad \Theta_{3}=U_{3} \frac{\omega^{2} m-E A \lambda_{2}^{2}}{K_{\mathrm{tt}} \lambda_{2}^{2}}, \quad \Theta_{4}=U_{4} \frac{\omega^{2} m-E A \lambda_{2}^{2}}{K_{\mathrm{tt}} \lambda_{2}^{2}} .
$$

End conditions are free, fixed, or mixed. At a free end, $T_{x}$ and $M_{x}$ are both zero, so conditions (8) and (9) become

$$
\left[\begin{array}{ll}
E A & K_{\mathrm{tt}} \\
K_{\mathrm{tt}} & G J
\end{array}\right]\left[\begin{array}{l}
U^{\prime} \\
\Theta^{\prime}
\end{array}\right]=\mathbf{0}
$$

Again, the determinant of the stiffness matrix in Eq. (18) cannot be equal to zero, so one must have $U^{\prime}=\Theta^{\prime}=0$. At a fixed end, one has simply $U=\Theta=0$. Two types of mixed end conditions are possible: if 
one allows extension but not rotation, then $\Theta=0$ and $U^{\prime}=-K_{\mathrm{tt}} \Theta^{\prime} /(E A)$; alternatively, if rotation is allowed, but not extension, then one has $U=0$ and $\Theta^{\prime}=-K_{\mathrm{tt}} U^{\prime} /(G J)$.

\subsection{Free-free end conditions}

For a free-free beam, application of the boundary condition at the end $x=0$ leads to the equations

$$
U_{1} \lambda_{1}+U_{3} \lambda_{2}=0, \quad \Theta_{1} \lambda_{1}+\Theta_{3} \lambda_{2}=0,
$$

which combined with Eq. (17), provides the condition

$$
\left[\begin{array}{cc}
\lambda_{1} & \lambda_{2} \\
\left(\omega^{2} m-E A \lambda_{1}^{2}\right) /\left(K_{\mathrm{tt}} \lambda_{1}\right) & \left(\omega^{2} m-E A \lambda_{2}^{2}\right) /\left(K_{\mathrm{tt}} \lambda_{2}\right)
\end{array}\right]\left[\begin{array}{l}
U_{1} \\
U_{3}
\end{array}\right]=\mathbf{0} .
$$

The determinant of the above can be zero only for the degenerate case $\lambda_{1}=\lambda_{2}$. However, the radical in Eq. (14) can be written as $\sqrt{\left(E A I_{x}-G J m\right)^{2}+4 m I_{x} K_{\mathrm{tt}}^{2}}$ which is intrinsically positive and could be zero only if $K_{\mathrm{tt}}=0$, and $E A / m=G J / I_{x}$. This would imply that one has uncoupled torsional and extensional vibration at the same frequency; instead, the relevant requirement is for $U_{1}=U_{3}=0$, and hence $\Theta_{1}=\Theta_{3}=0$. Application of the boundary conditions at the end $x=L$, leads to the frequency equations

$$
\sin \lambda_{1} L=0, \quad \sin \lambda_{2} L=0
$$

and setting $\lambda_{1} L=n \pi, \quad \lambda_{2} L=n \pi, \quad n=1,2,3, \ldots$, etc., Eq. (15) leads to the natural frequency predictions: predominantly extensional:

$$
\omega_{1}=\frac{n \pi}{L} \sqrt{\frac{E A I_{x}+G J m+\sqrt{\left(E A I_{x}-G J m\right)^{2}+4 m I_{x} K_{\mathrm{tt}}^{2}}}{2 m I_{x}}},
$$

predominantly torsional:

$$
\omega_{2}=\frac{n \pi}{L} \sqrt{\frac{E A I_{x}+G J m-\sqrt{\left(E A I_{x}-G J m\right)^{2}+4 m I_{x} K_{\mathrm{tt}}^{2}}}{2 m I_{x}}}
$$

with mode shapes

$$
U(x)=U_{2} \cos \frac{n \pi x}{L}, \quad \Theta(x)=\Theta_{2} \cos \frac{n \pi x}{L}, \quad n=1,2,3, \ldots, \text { etc. }
$$

It is noted that Eqs. (22) and (23) are equally applicable to fixed-fixed end conditions, but the mode shapes are then sinusoidal rather than cosinusoidal. It is not immediately obvious which of Eqs. (22), (23) should be described as predominantly torsional or extensional, other than the expectation that the predominantly torsional frequency will be the lower of the two, and therefore should be associated with the minus sign; however, the above do reduce to the familiar expressions for uncoupled torsional and extensional vibration, which are $\omega_{T}=(n \pi / L) \sqrt{G J / I_{x}}$ and $\omega_{E}=(n \pi / L) \sqrt{E A / m}$, respectively, when one sets $K_{\mathrm{tt}}=0$, and their nature has been confirmed through FEM animations for the considered structures. In the appendix, it is shown that these designations are indeed correct, so long as the uncoupled extensional frequency is greater than the uncoupled torsional frequency. Indeed, whichever mode has the lower frequency when uncoupled continues to have the lower frequency when coupled.

\subsection{Fixed-free end conditions}

For a fixed-free beam, one finds from the conditions at $x=0$

$$
U_{2}=U_{4}=\Theta_{2}=\Theta_{4}=0,
$$


while the conditions at $x=L$ lead to the frequency equations

$$
\cos \lambda_{1} L=0, \quad \cos \lambda_{2} L=0
$$

and hence the frequency predictions are:

predominantly extensional:

$$
\omega_{1}=\frac{n \pi}{2 L} \sqrt{\frac{E A I_{x}+G J m+\sqrt{\left(E A I_{x}-G J m\right)^{2}+4 m I_{x} K_{\mathrm{tt}}^{2}}}{2 m I_{x}}},
$$

predominantly torsional:

$$
\omega_{2}=\frac{n \pi}{2 L} \sqrt{\frac{E A I_{x}+G J m-\sqrt{\left(E A I_{x}-G J m\right)^{2}+4 m I_{x} K_{\mathrm{tt}}^{2}}}{2 m I_{x}}}
$$

with modes shapes

$$
U(x)=U_{1} \sin \frac{n \pi x}{2 L}, \quad \Theta(x)=\Theta_{1} \sin \frac{n \pi x}{2 L}, \quad n=1,3,5, \ldots, \text { etc. }
$$

\section{Comparison of natural frequency predictions}

First, consider the coupled torsion-extension predictions, Table 1, for a long (30 cells) asymmetric structure, having a total length $L=10.284 \mathrm{~m}$, assuming free-free and fixed-fixed end conditions. Better agreement with the FEM predictions arises when the latter employs the consistent mass matrix, and only these are shown. The results show excellent agreement; for the predominantly torsional modes, the accuracy is within $+0.02 \%$ to $+1.30 \%$ for the free-free beam, and $\pm 0.65 \%$ for the fixed-fixed beam, for the first 9 modes. For the first 2 extensional modes, the maximum error is $-0.05 \%$ and $-0.33 \%$ for free-free and fixed-fixed beams, respectively. Also note that the FEM predictions for the fixed-fixed case are slightly larger than those for the free-free, by a fairly consistent $0.7 \%$; on the other hand, the equivalent continuum predictions are identical, the two cases having identical frequency equations. Most likely, this difference arises because the FEM is able to model local effects at a fixed end, e.g. the suppression of Poisson's ratio effects, while the equivalent continuum model cannot; in turn, additional constraint implies greater stiffness, and hence higher natural frequency. One might argue, therefore, that comparison with the free-free case is the more valid of the two.

\begin{tabular}{|c|c|c|c|c|c|c|c|}
\hline \multicolumn{4}{|c|}{ Free-free beam } & \multicolumn{4}{|c|}{ Fixed-fixed beam } \\
\hline$n$ & FEM & Torsional & Extensional & $n$ & FEM & Torsional & Extensional \\
\hline 1 & 37.58 & $37.59(+0.02 \%)$ & & 1 & 37.83 & $37.59(-0.65 \%)$ & \\
\hline 2 & 75.11 & $75.17(+0.08 \%)$ & & 2 & 75.62 & $75.17(-0.59 \%)$ & \\
\hline 3 & 112.55 & $112.76(+0.18 \%)$ & & 3 & 113.31 & $112.76(-0.49 \%)$ & \\
\hline 4 & 149.86 & $150.34(+0.32 \%)$ & & 4 & 150.89 & $150.34(-0.36 \%)$ & \\
\hline 5 & 151.81 & & $151.79(-0.02 \%)$ & 5 & 152.12 & & $151.79(-0.22 \%)$ \\
\hline 6 & 186.98 & $187.93(+0.51 \%)$ & & 6 & 188.30 & $187.93(-0.20 \%)$ & \\
\hline 7 & 223.88 & $225.51(+0.73 \%)$ & & 7 & 225.46 & $225.51(+0.02 \%)$ & \\
\hline 8 & 260.51 & $263.10(+0.99 \%)$ & & 8 & 262.39 & $263.10(+0.27 \%)$ & \\
\hline 9 & 296.82 & $300.69(+1.30 \%)$ & & 9 & 298.80 & $300.69(+0.63 \%)$ & \\
\hline 10 & 303.73 & & $303.57(-0.05 \%)$ & 10 & 304.59 & & $303.57(-0.33 \%)$ \\
\hline 11 & 332.77 & $332.22(-0.92 \%)$ & & 11 & 335.29 & $332.22(+1.25 \%)$ & \\
\hline
\end{tabular}

Table 1

Comparison of natural frequencies $(\mathrm{Hz})$ in torsion and extension for asymmetric beam; free-free and fixed-fixed end conditions, $L=$ $10.284 \mathrm{~m}$ ( 30 cells); $n$ is here the mode number 
Table 2

Comparison of natural frequencies $(\mathrm{Hz})$ in torsion and extension for asymmetric beam; free-free and fixed-fixed end conditions, $L=$ $3.428 \mathrm{~m}$ ( 10 cells); $n$ is the mode number

\begin{tabular}{|c|c|c|c|c|c|c|c|}
\hline \multicolumn{4}{|c|}{ Free-free beam } & \multicolumn{4}{|c|}{ Fixed-fixed beam } \\
\hline$n$ & FEM & Torsional & Extensional & $n$ & FEM & Torsional & Extensional \\
\hline 1 & 112.52 & $112.76(+0.21 \%)$ & & 1 & 114.84 & $112.76(-1.81 \%)$ & \\
\hline 2 & 223.64 & $225.51(+0.84 \%)$ & & 2 & 228.46 & $225.51(-1.29 \%)$ & \\
\hline 3 & 331.92 & $338.27(+1.91 \%)$ & & 3 & 339.42 & $338.27(-0.34 \%)$ & \\
\hline 4 & 435.82 & $451.03(+3.49 \%)$ & & 4 & 446.74 & $451.03(+0.96 \%)$ & \\
\hline 5 & 455.84 & & $455.36(-0.11 \%)$ & 5 & 458.85 & & $455.36(-0.76 \%)$ \\
\hline 6 & 533.53 & $563.79(+5.67 \%)$ & & 6 & 548.67 & $563.79(+2.76 \%)$ & \\
\hline 7 & 622.63 & $676.54(+7.97 \%)$ & & 7 & 641.72 & $676.54(+5.43 \%)$ & \\
\hline
\end{tabular}

Table 3

Comparison of natural frequencies $(\mathrm{Hz})$ in torsion and extension for asymmetric beam; fixed-free end conditions; $n$ is the mode number

\begin{tabular}{|c|c|c|c|c|c|c|c|}
\hline \multicolumn{4}{|c|}{ 10-cell structure } & \multicolumn{4}{|c|}{ 30-cell structure } \\
\hline$n$ & FEM & Torsional & Extensional & $n$ & FEM & Torsional & Extensional \\
\hline 1 & 56.92 & $56.38(-0.95 \%)$ & & 1 & 18.86 & $18.79(-0.33 \%)$ & \\
\hline 2 & 170.08 & $169.14(-0.56 \%)$ & & 2 & 56.54 & $56.38(-0.29 \%)$ & \\
\hline 3 & 228.46 & & $227.68(-0.34 \%)$ & 3 & 75.97 & & $75.89(-0.11 \%)$ \\
\hline 4 & 281.30 & $281.89(+0.21 \%)$ & & 4 & 94.16 & $93.96(-0.21 \%)$ & \\
\hline 5 & 389.06 & $394.65(+1.44 \%)$ & & 5 & 131.67 & $131.55(-0.09 \%)$ & \\
\hline 6 & 491.81 & $507.41(+3.17 \%)$ & & 6 & 169.02 & $169.14(+0.07 \%)$ & \\
\hline 7 & 587.52 & $620.17(+5.56 \%)$ & & 7 & 206.17 & $206.72(+0.27 \%)$ & \\
\hline 8 & 672.36 & $732.92(+9.01 \%)$ & & 8 & 227.98 & & $227.68(-0.13 \%)$ \\
\hline 9 & 688.20 & & $688.04(-0.75 \%)$ & 9 & 243.11 & $244.31(+0.49 \%)$ & \\
\hline 10 & 745.46 & $845.68(+13.44 \%)$ & & 10 & 279.72 & $281.89(+0.78 \%)$ & \\
\hline
\end{tabular}

For the short (10 cell) beam, Table 2, good agreement is obtained up to the fourth torsional mode for free-free $(+0.21 \%$ to $+3.49 \%)$ and up to the sixth torsional mode for fixed-fixed beam end conditions $(-1.81 \%$ to $+2.76 \%)$; again the agreement for the extensional modes is excellent. Again the FEM predictions are greater for fixed-fixed conditions, by some $2-3 \%$ for the torsional modes, and by $0.7 \%$ for the extensional. Similar accuracy and trends for the natural frequency agreement can be seen in Table 3, for both the long and short fixed-free beams.

Predictions for the pre-twisted structure, with $\alpha=\pi / 8$, are given in Tables 4 and 5. For the long, 30 cell, beam with both free-free and fixed-free end conditions, the accuracy range is $-0.04 \%$ to $+3.15 \%$ for the first 10 torsional modes, and within $-0.31 \%$ for the first 4 extensional modes. For the short, 10 cell beam, Table 5 , there is again excellent agreement for the 2 lowest extensional modes, while the first 4 torsional modes are within $+4.75 \%$.

Last, Figs. 3-5 show the fundamental torsional and extensional natural frequencies for a short (10 cells) structure, with both free-free and fixed-free end conditions, for pre-twist angle per cell ranging from $0^{\circ}$ to $180^{\circ}$. It is seen that the torsional frequencies are, in general, much more sensitive to pre-twist, than the extensional frequencies. For pre-twist up to about $80^{\circ}$ per cell, torsional frequencies are increased by approximately the same amount as extensional frequencies are depressed; however, the percentage changes are much greater for the former. From Figs. 3 and 4, it is clear that agreement between FEM and the equivalent continuum model predictions is excellent over this wide range of pre-twist angles. The differences are shown in Fig. 5, where it is seen that the continuum model for the predominantly torsional modes is most often greater than the FEM predictions, while those of the predominantly extensional 
Table 4

Comparison of natural frequencies $(\mathrm{Hz})$ in torsion and extension for pre-twisted beam; free-free and fixed-free end conditions, $L=$ $10.284 \mathrm{~m}$ (30 cells $) ; n$ is the mode number

\begin{tabular}{|c|c|c|c|c|c|c|c|}
\hline \multicolumn{4}{|c|}{ Free-free beam } & \multicolumn{4}{|c|}{ Fixed-free beam } \\
\hline$n$ & FEM & Torsional & Extensional & $n$ & FEM & Torsional & Extensional \\
\hline 1 & 56.41 & $56.42(+0.03 \%)$ & & 1 & 28.22 & $28.21(-0.04 \%)$ & \\
\hline 2 & 112.71 & $112.84(+0.12 \%)$ & & 2 & 73.73 & & $73.64(-0.13 \%)$ \\
\hline 3 & 147.30 & & $147.27(-0.02 \%)$ & 3 & 84.62 & $84.63(+0.02 \%)$ & \\
\hline 4 & 168.82 & $169.30(+0.26 \%)$ & & 4 & 140.86 & $141.05(+0.14 \%)$ & \\
\hline 5 & 224.62 & $225.69(+0.47 \%)$ & & 5 & 196.85 & $197.48(+0.32 \%)$ & \\
\hline 6 & 280.02 & $282.11(+0.74 \%)$ & & 6 & 221.26 & & $220.91(-0.16 \%)$ \\
\hline 7 & 294.73 & & $294.55(-0.06 \%)$ & 7 & 252.50 & $253.90(+0.55 \%)$ & \\
\hline 8 & 334.91 & $338.53(+1.08 \%)$ & & 8 & 307.68 & $310.32(+0.86 \%)$ & \\
\hline 9 & 389.17 & $394.95(+1.49 \%)$ & & 9 & 362.28 & $366.74(+1.23 \%)$ & \\
\hline 10 & 442.44 & & $441.81(-0.14 \%)$ & 10 & 369.01 & & $368.18(-0.22 \%)$ \\
\hline 11 & 442.68 & $451.37(+1.96 \%)$ & & 11 & 416.22 & $423.16(+1.67 \%)$ & \\
\hline 12 & 495.33 & $507.79(+2.52 \%)$ & & 12 & 469.34 & $479.58(+2.18 \%)$ & \\
\hline 13 & 546.99 & $564.22(+3.15 \%)$ & & 13 & 517.07 & & $515.46(-0.31 \%)$ \\
\hline 14 & 590.54 & & $589.10(-0.24 \%)$ & 14 & 521.56 & $536.01(+2.77 \%)$ & \\
\hline
\end{tabular}

Table 5

Comparison of natural frequencies $(\mathrm{Hz})$ in torsion and extension of pre-twisted beam; free-free and fixed-free end conditions, $L=3.428 \mathrm{~m}$ (10 cells); $n$ is the mode number

\begin{tabular}{|c|c|c|c|c|c|c|c|}
\hline \multicolumn{4}{|c|}{ Free-free beam } & \multicolumn{4}{|c|}{ Fixed-free beam } \\
\hline$n$ & FEM & Torsional & Extensional & $n$ & FEM & Torsional & Extensional \\
\hline 1 & 168.81 & $169.25(+0.26 \%)$ & & 1 & 84.69 & $84.63(-0.07 \%)$ & \\
\hline 2 & 334.85 & $338.53(+1.10 \%)$ & & 2 & 221.79 & & $220.91(-0.40 \%)$ \\
\hline 3 & 442.39 & & $441.82(-0.13 \%)$ & 3 & 252.71 & $253.90(+0.47 \%)$ & \\
\hline 4 & 495.15 & $507.79(+2.55 \%)$ & & 4 & 416.98 & $423.16(+1.60 \%)$ & \\
\hline 5 & 646.36 & $677.06(+4.75 \%)$ & & 5 & 572.92 & $592.43(+3.41 \%)$ & \\
\hline 6 & 784.72 & $846.32(+7.85 \%)$ & & 6 & 667.21 & & $662.73(-0.67 \%)$ \\
\hline 7 & 887.87 & & $883.64(-0.48 \%)$ & 7 & 718.47 & $761.69(+6.02 \%)$ & \\
\hline
\end{tabular}

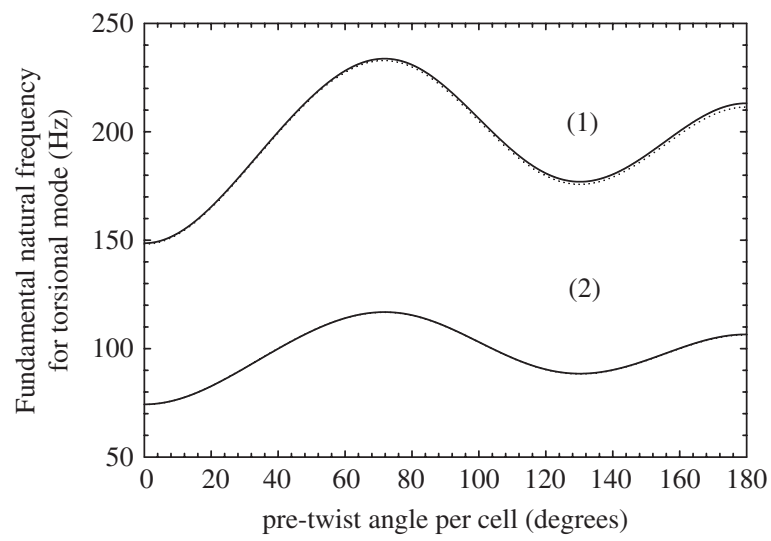

Fig. 3. Variation of fundamental predominantly torsional natural frequency with angle of pre-twist per cell. The upper curves (1) are for free-free end conditions; the solid line pertains to the equivalent continuum model, while the dotted line is the FEM prediction. The lower curve (2) is for fixed-free end conditions; the differences between the equivalent continuum and FEM predictions are not discernable. 


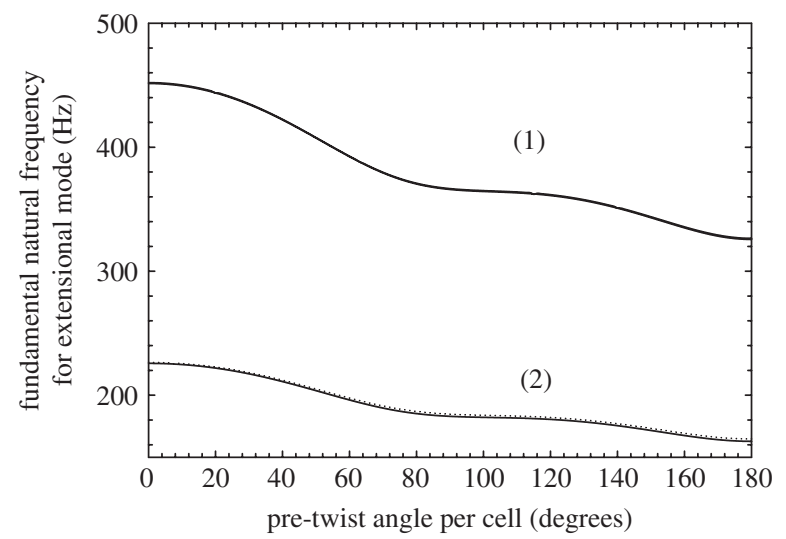

Fig. 4. Variation of fundamental predominantly extensional natural frequency with angle of pre-twist per cell. The upper curve (1) is for free-free end conditions; the differences between the equivalent continuum and FEM predictions are not discernable. The lower curves (2) are for fixed-free end conditions; the solid line pertains to the equivalent continuum model, while the dotted line is the FEM prediction.

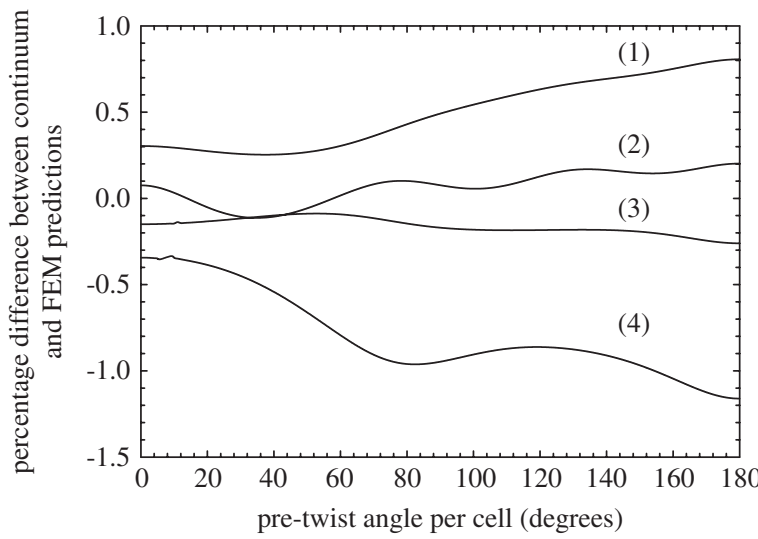

Fig. 5. Percentage difference between equivalent continuum and FEM predictions ((equivalent continuum-FEM)/FEM $\times 100 \%)$ for the fundamental predominantly torsional and extensional natural frequencies with pre-twist angle. From top to bottom, the curves are (1) for torsional (free-free), (2) torsional (clamped-free), (3) extensional (free-free) and (4) extensional (fixed-free).

modes are lower than the corresponding finite element predictions. However, the errors for both are very small.

\section{Concluding remarks}

The simplest dynamic model for tension-torsion coupling of beam structures, due to Di Prima [22], has been extended to more general beam end conditions. The model ignores higher-order effects, such as Poisson's ratio contraction during extensional vibration (see Love [26]), and axial warping effects during torsion for noncircular cross-sections (see [27] for a review and comparison of several theories), but is seen to be quite adequate for prediction of the lower frequencies of vibration of the two structure types considered. Indeed, since one should not expect an equivalent continuum model to adequately describe the dynamic behaviour of a discrete structure as semi-wavelength becomes as small as the length of individual members - when local resonances would occur - it would seem that these higher-order effects can be ignored, at least for the coupled tension-torsion considered here. The same conclusion was drawn in [19] for uncoupled extensional vibration of a planar structure but, in contrast, it was seen that higher-order shear and Timoshenko models were required for accurate prediction of even the lowest modes of flexural vibration. 


\section{Appendix A}

Here it is shown that whichever mode has the lower uncoupled natural frequency continues to have the lower frequency when coupled. In general, this will be the predominantly torsional mode. From Eq. (17b), with $\lambda_{1}$ set equal to $n \pi / L$, one has

$$
\frac{U_{2}}{\theta_{2}}=\frac{K_{\mathrm{tt}}(n \pi / L)^{2}}{m\left(\omega^{2}-(E A / m)(n \pi / L)^{2}\right)}=\frac{K_{\mathrm{tt}}(n \pi / L)^{2}}{m\left(\omega^{2}-\omega_{E}^{2}\right)} .
$$

From Eqs. (22), (23), the squares of the frequency may be written as

$$
\omega_{1,2}^{2}=\left(\frac{n \pi}{L}\right)^{2} \frac{E A I_{x}+G J m \pm \sqrt{\left(E A I_{x}-G J m\right)^{2}+4 m I_{x} K_{\mathrm{tt}}^{2}}}{2 m I_{x}}
$$

or in terms of the uncoupled frequencies

$$
\omega_{1,2}^{2}=\frac{\omega_{T}^{2}}{2}+\frac{\omega_{E}^{2}}{2} \pm \sqrt{\left(\frac{\omega_{T}^{2}}{2}-\frac{\omega_{E}^{2}}{2}\right)^{2}+\Delta},
$$

where $\Delta=(n \pi / L)^{4} K_{\mathrm{tt}}^{2} /\left(m I_{x}\right)$ and is obviously positive. Substituting into Eq. (A.1) gives

$$
\frac{U_{2}}{\theta_{2}}=\frac{2 K_{\mathrm{tt}}(n \pi / L)^{2}}{m\left(\omega_{T}^{2}-\omega_{E}^{2} \pm \sqrt{\left(\omega_{T}^{2}-\omega_{E}^{2}\right)^{2}+4 \Delta}\right)} .
$$

Now, under the assumption that the uncoupled extensional frequency is greater than the uncoupled torsional, i.e. $\omega_{E}^{2}>\omega_{T}^{2}$, write $\beta=\omega_{E}^{2}-\omega_{T}^{2}$, where $\beta$ is positive. Eq. (A.4) can now be written as

$$
\frac{U_{2}}{\theta_{2}}=\frac{-2 K_{\mathrm{tt}}(n \pi / L)^{2}}{m\left(\beta \mp \sqrt{\beta^{2}+4 \Delta}\right)},
$$

where one must note that the positive and minus signs associated with Eq. (A.4) are now reversed. Further note that the radical in Eq. (A.5) may be expressed as $\sqrt{\beta^{2}+4 \Delta}=\beta+\varepsilon$, where $\varepsilon$ must also be positive, leading to

$$
\frac{U_{2}}{\theta_{2}}=\frac{-2 K_{\mathrm{tt}}(n \pi / L)^{2}}{m(\beta \mp(\beta+\varepsilon))} .
$$

For the original positive sign in Eq. (A.3) leading to the higher frequency, $\omega_{1}^{2}$, one must employ the negative sign in Eq. (A.6), to give

$$
\left(\frac{U_{2}}{\theta_{2}}\right)_{1}=\frac{2 K_{\mathrm{tt}}(n \pi / L)^{2}}{m \varepsilon} .
$$

On the other hand, for the original negative sign in Eq. (A.3) leading to the lower frequency, $\omega_{2}^{2}$, one must employ the positive sign in Eq. (A.6), to give

$$
\left(\frac{U_{2}}{\theta_{2}}\right)_{2}=\frac{-2 K_{\mathrm{tt}}(n \pi / L)^{2}}{m(2 \beta+\varepsilon)} .
$$

Since the possibility of $\beta=0$ has been discounted previously, the magnitude of $\left(U_{2} / \theta_{2}\right)_{1}$ is clearly larger than the magnitude of $\left(U_{2} / \theta_{2}\right)_{2}$, and one may conclude that the predominantly extensional and torsional modes are associated with the original positive and negative signs, respectively, within Eqs. (22), (23) or, equivalently, with the subscripts 1 and 2 in Eqs. (A.7), (A.8). From Eqs. (A.7), (A.8) it is clear that extension and rotation are of opposite phase for the two modes; for the pre-twisted structure, since $\left(U_{2} / \theta_{2}\right)_{1}$ is negative (recall that $K_{\mathrm{tt}}$ is negative), large extension is coupled with a small rotation tending to increase the initial pre-twist. On the 
other hand, since $\left(U_{2} / \theta_{2}\right)_{2}$ is positive, large rotation tending to increase the initial pre-twist leads to axial contraction. The above argument assumes that the uncoupled torsional frequency is lower than the uncoupled extensional, which is what one would expect for a continuum structure. In principle one could construct a reticulated structure for which the opposite is true; the above reasoning is then repeated under the assumption that $\beta=\omega_{T}^{2}-\omega_{E}^{2}$, and one would conclude that the greater frequency is associated with the predominantly torsional coupled mode. Overall, one concludes that the lower (higher) of the two coupled frequencies has the same predominant character as that of the lower (higher) uncoupled frequency.

\section{References}

[1] A.K. Noor, Continuum modelling for repetitive lattice structures, Applied Mechanics Reviews 41 (1998) $285-296$.

[2] D.L. Dean, Discrete Field Analysis of Structural Systems, Springer, New York, 1976.

[3] J.D. Renton, The beam-like behaviour of space trusses, AIAA Journal 22 (1984) 273-280.

[4] D.J. Mead, Free wave propagation in periodically supported infinite beams, Journal of Sound and Vibration 13 (1970) $181-197$.

[5] L. Meirowitz, R.C. Engels, Response of periodic structures by the z-transform method, AIAA Journal 15 (1977) $167-174$.

[6] Y. Yong, Y.K. Lin, Propagation of decaying waves in periodic and piecewise periodic structures of finite length, Journal of Sound and Vibration 129 (1989) 98-118.

[7] Y. Yong, Y.K. Lin, Dynamics of complex truss-type space structures, AIAA Journal 28 (1989) 1250-1258.

[8] W.X. Zhong, F.W. Williams, Wave problems for repetitive structures and symplectic mathematics, Journal of Mechanical Engineering Science 206 (1992) 371-379.

[9] W.X. Zhong, F.W. Williams, On the direct solution of wave propagation for repetitive structures, Journal of Sound and Vibration 181 (1995) 485-501.

[10] D.J. Mead, Wave propagation in continuous periodic structures: research contributions from Southampton, Journal of Sound and Vibration 190 (1996) 495-520.

[11] R.S. Langley, A transfer matrix analysis of the energetics of structural wave motion and harmonic vibration, Proceeding of the Royal Society, Series A 452 (1996) 1631-1648.

[12] N.G. Stephen, P.J. Wang, On Saint-Venant's principle in pin-jointed frameworks, International Journal of Solids and Structures 33 (1996) 79-96.

[13] N.G. Stephen, Y. Zhang, Eigenanalysis and continuum modelling of an asymmetric beam-like repetitive structure, International Journal of Mechanical Sciences 46 (2004) 1213-1231.

[14] N.G. Stephen, S. Ghosh, Eigenanalysis and continuum modelling of a curved repetitive beam-like structure, International Journal of Mechanical Sciences 47 (2005) 1854-1873.

[15] N.G. Stephen, Y. Zhang, Eigenanalysis and continuum modelling of pre-twisted repetitive beam-like structures. International Journal of Solids and Structures, in press.

[16] R.S. Langley, N.S. Bardell, A review of current analysis capabilities applicable to the high frequency vibration prediction of aerospace structures, The Aeronautical Journal 102 (1998) 287-297.

[17] K.U. Machens, I. Dyer, Energy partitioning in a truss structure, Acustica 81 (1995) 587-594.

[18] R.S. Langley, Mid and high-frequency vibration analysis of structures with uncertain properties. Eleventh International Congress on Sound and Vibration, July 2004, St. Petersburg.

[19] N.G. Stephen, On the vibration of one-dimensional periodic structures, Journal of Sound and Vibration 227 (1999) $1133-1142$.

[20] A. Rosen, Structural and dynamic behaviour of pretwisted rods and beams, ASME Applied Mechanics Reviews 44 (1991) $483-515$.

[21] E. Reissner, K. Washizu, On torsional vibrations of a beam with a small amount of pretwist, Journal of the Japan Society of Aeronautical Engineers 5 (1957) 330-335.

[22] R.C. Di Prima, Coupled torsional and longitudinal vibrations of a thin bar, ASME Journal of Applied Mechanics 26 (1959) 510-512.

[23] C. Chen, The effect of initial twist on the torsional rigidity of thin prismatic bars and tubular members, Proceedings of the First US National Congress of Applied Mechanics, 1951, pp. 265-269.

[24] http://images.jsc.nasa.gov/index.html The official NASA photograph ID is STS61B-120-052.

[25] C.S. Desai, J.F. Abel, Introduction to the Finite Element Method, Van Nostrand Reinhold, New York, 1972.

[26] A.E.H. Love, A Treatise on the Mathematical Theory of Elasticity, fourth ed., Dover, New York, 1944 article 278.

[27] N.G. Stephen, Comparison of dynamic torsion theories for beams of elliptical cross-section, Journal of Sound and Vibration 100 (1985) $1-6$. 